\title{
BASES IN THE SOLUTION SPACE OF THE MELLIN SYSTEM
}

\author{
ALICIA DICKENSTEIN AND TIMUR SADYKOV
}

ABStRACt. We consider algebraic functions $z$ satisfying equations of the form

$$
a_{0} z^{m}+a_{1} z^{m_{1}}+a_{2} z^{m_{2}}+\ldots+a_{n} z^{m_{n}}+a_{n+1}=0 .
$$

Here $m>m_{1}>\ldots>m_{n}>0, m, m_{i} \in \mathbb{N}$, and $z=z\left(a_{0}, \ldots, a_{n+1}\right)$ is a function of the complex variables $a_{0}, \ldots, a_{n+1}$. Solutions to such equations are classically known to satisfy holonomic systems of linear partial differential equations with polynomial coefficients. In this paper we investigate one of such systems of differential equations which was introduced by Mellin. We compute the holonomic rank of the Mellin system as well as the dimension of the space of its algebraic solutions. Moreover, we construct explicit bases of solutions in terms of the roots of (0.1) and their logarithms. We show that the monodromy of the Mellin system is always reducible and give some factorization results in the univariate case.

\section{INTRODUCTION}

Consider the class of algebraic functions which satisfy algebraic equations with symbolic coefficients, i.e., equations of the form

$$
a_{0} z^{m}+a_{1} z^{m_{1}}+a_{2} z^{m_{2}}+\ldots+a_{n} z^{m_{n}}+a_{n+1}=0 .
$$

Here $m>m_{1}>\ldots>m_{n}>0, m, m_{i} \in \mathbb{N}, z=z\left(a_{0}, \ldots, a_{n+1}\right)$ is a function of the complex variables $a_{0}, \ldots, a_{n+1}$. Any solution to (1.1) satisfies the $A$-hypergeometric system defined by Gelfand, Kapranov and Zelevinsky [8] (see also [16, 11]) associated to the matrix

$$
A=\left(\begin{array}{ccccc}
1 & 1 & \ldots & 1 & 1 \\
m & m_{1} & \ldots & m_{n} & 0
\end{array}\right)
$$

and the homogeneity vector $(0,-1)$. In particular, a solution to (1.1) has a double homogeneity property and can therefore be considered as a function of $n$ variables. This implies that we can arbitrarily prescribe the values of any two nonzero coefficients in (1.1) without losing any essential information on the general solution to this equation. It turns out to be convenient to divide (1.1) by $-a_{n+1}$ and then set $y=\left(-a_{0} / a_{n+1}\right)^{1 / m} z$, reducing (1.1) to an equation of the form

$$
y^{m}+x_{1} y^{m_{1}}+\ldots+x_{n} y^{m_{n}}-1=0 .
$$

A classical result of Mellin from 1921 (see [10]) states that solutions to such equations satisfy certain systems of partial differential equations of hypergeometric type. More

Alicia Dickenstein was partially supported by UBACYT X042, CONICET PIP 5617 and ANPCYT PICT 20569, Argentina.

Timur Sadykov was partially supported by the Russian Foundation for Basic Research, grant 05-01-00517 and by the grant MK-851.2006.1 of the President of Russian Federation. 
precisely, if $y(x)=y\left(x_{1}, \ldots, x_{n}\right)$ is a solution to (1.2), then it satisfies the following system of $n$ partial differential equations:

$$
\begin{aligned}
& \prod_{k=0}^{m_{j}-1}\left(m_{1} \theta_{1}+\ldots+m_{n} \theta_{n}+m k+1\right) \prod_{k=0}^{m_{j}^{\prime}-1}\left(m_{1}^{\prime} \theta_{1}+\ldots+m_{n}^{\prime} \theta_{n}+m k-1\right) y(x)= \\
& (-1)^{m_{j}} m^{m} \frac{\partial^{m} y(x)}{\partial x_{j}^{m}}, \quad j=1, \ldots, n,
\end{aligned}
$$

where $\theta_{j}=x_{j} \frac{\partial}{\partial x_{j}}$ and $m_{j}^{\prime}=m-m_{j}$. In this article we will only consider algebraic equations of the form (1.2). The system (1.3) will be referred to as the Mellin system of equations associated with (1.2).

The goal of this paper is to describe all the solutions of the Mellin system of equations and to construct explicit bases of local complex analytic solutions for any $n$ and any value of the greatest common divisor $d$ of $m, m_{1}, \ldots, m_{n}$. We describe the space of algebraic solutions and we exhibit explicit non-algebraic solutions in terms of the roots of (1.2) and their logarithms. These problems arose in the framework of the general theory of hypergeometric functions and their relations with algebraic equations, see [4], 6], [13, [16], and [17].

The special case of the trinomial equation $y^{m}+x y^{p}-1=0$ (which corresponds to ordinary Mellin equation) was thoroughly studied in [9]. The Mellin equation is always reducible and unless $p=m-1$, it is never the linear differential equation with polynomial coefficients of smallest order satisfied by the roots of the trinomial equation. Moreover, in this case all solutions to the Mellin equation are algebraic except in the case when $d=1$ and $p<m-1$, where we exhibit an explicit nonalgebraic solution, as a special case of our main Theorem 4.3 .

We show that the monodromy of the Mellin equations is always reducible. This could be deduced when $d=n=1$ from the general results in [17] since in this case the homogeneity $\beta$ has integer coordinates. In the last section, we give some factorization results in the univariate case.

Acknowledgements: The authors are grateful to Martín Mereb for useful comments on Theorem 4.3 and to Michael Singer for interesting discussions and for his help in the proof of Proposition 5.5. T. Sadykov is greatly indebted to his colleagues and staff at the Max-Planck Institut für Mathematik in Bonn for creating a wonderful working atmosphere.

\section{Convenient Bases And Generating solutions of the Mellin system}

Mellin calls the solution of (1.2) which assumes the value 1 for $x_{1}=\ldots=x_{n}=0$ the principal solution of this equation. He shows (see [10]) that the power series expansion of the principal solution to (1.2) around the origin is given by

$$
y_{p r}(x)=\sum_{\nu_{1}, \ldots, \nu_{n} \geq 0} \frac{(-1)^{|\nu|}}{m^{|\nu|}} \frac{\prod_{\mu=1}^{|\nu|-1}\left(m_{1} \nu_{1}+\ldots+m_{n} \nu_{n}-m \mu+1\right)}{\nu_{1} ! \ldots \nu_{n} !} x_{1}^{\nu_{1}} \ldots x_{n}^{\nu_{n}} .
$$

Here $|\nu|=\nu_{1}+\ldots+\nu_{n}$ and the empty product is defined to be 1 . 
For a multi-index $I=\left(i_{1}, \ldots, i_{n}\right)$ and a complex vector $x=\left(x_{1}, \ldots, x_{n}\right) \in \mathbb{C}^{n}$ we use the standard notation $x^{I}=x_{1}^{i_{1}} \ldots x_{n}^{i_{n}}$. By $B$ we denote the set

$$
B=\left\{\left(i_{1}, \ldots, i_{n}\right) \in \mathbb{Z}^{n}: 0 \leq i_{j} \leq m-1, \text { for any } j=1, \ldots, n\right\} .
$$

Theorem 2.1. The dimension of the space of analytic solutions to (1.3) at a generic point, i.e., the holonomic rank of the Mellin system, equals $m^{n}$. There exists a basis $\left\{f_{I}\right\}_{I \in B}$ around the origin such that

$$
f_{I}(x)=x^{I} \tilde{f}_{I}\left(x_{1}^{m}, \ldots, x_{n}^{m}\right)
$$

where $\tilde{f}_{I}$ is analytic at 0 and $\tilde{f}_{I}(0) \neq 0$ for any $I \in B$.

We say that a system of functions of the form (2.2) forms a convenient basis in the solution space of the Mellin system (1.3).

The proof of this theorem can be deduced from Theorems 2.8 and 3.1 in [14] by adjusting the arguments in [14] to the case of a nonconfluent hypergeometric system. For the benefit of the reader we give now a proof which uses the results of [14] as little as possible. We will reprove a part of this theorem in the next section, as a consequence of the relation with the $A$-hypergeometric system associated to the exponents $m, m_{1}, \ldots, m_{n}, 0$, and its translation to a Horn system [6].

Proof. For the sake of brevity we will use the notation $M=\left(m_{1}, \ldots, m_{n}\right), M^{\prime}=$ $\left(m_{1}^{\prime}, \ldots, m_{n}^{\prime}\right), \theta=\left(\theta_{1}, \ldots, \theta_{n}\right)$ and denote by $\langle\cdot, \cdot\rangle$ the scalar product. We multiply the $j$-th equation in (1.3) with $x_{j}^{m}$. This will not affect the space of holomorphic solutions to (1.3) . Let us denote by $P_{j}(\theta)$ the polynomial $\prod_{k=0}^{m_{j}-1}(\langle M, \theta\rangle+m k+$ 1) $\prod_{k=0}^{m_{j}^{\prime}-1}\left(\left\langle M^{\prime}, \theta\right\rangle+m k-1\right)$ and by $G_{j}$ the differential operator

$$
G_{j}=x_{j}^{m} P_{j}(\theta)-(-1)^{m_{j}} m^{m} x_{j}^{m} \frac{\partial^{m}}{\partial x_{j}^{m}}, j=1, \ldots, n .
$$

Let $\mathcal{D}$ be the Weyl algebra in $n$ variables [3], and define $\mathcal{M}=\mathcal{D} / \sum_{j=1}^{n} \mathcal{D} G_{j}$ to be the left $\mathcal{D}$-module associated with the system of differential operators (2.3). Let $R=\mathbb{C}\left[z_{1}, \ldots, z_{n}\right]$ and $R[x]=R\left[x_{1}, \ldots, x_{n}\right]=\mathbb{C}\left[x_{1}, \ldots, x_{n}, z_{1}, \ldots, z_{n}\right]$. We make $R[x]$ into a left $\mathcal{D}$-module by defining the action of $\partial_{j}$ on $R[x]$ by

$$
\partial_{j}=\frac{\partial}{\partial x_{j}}+z_{j}
$$

Let us define the operators $D_{j}: R[x] \rightarrow R[x]$ by setting

$$
D_{j}=z_{j} \frac{\partial}{\partial z_{j}}+x_{j} z_{j}, \quad j=1, \ldots, n \text {. }
$$

It was pointed out in [1] that the operators (2.5) form a commutative family of $\mathcal{D}$-linear operators. Let $D$ denote the vector $\left(D_{1}, \ldots, D_{n}\right)$. For any $j=1, \ldots, n$ we define the operator $\nabla_{j}: R[x] \rightarrow R[x]$ by $\nabla_{j}=z_{j}^{-1} D_{j}$. This operator commutes with the operators $\partial_{k}$ since both $D_{j}$ and the multiplication by $z_{j}^{-1}$ commute with $\partial_{k}$. Moreover, the operator $\nabla_{j}$ commutes with $\nabla_{k}$ for all $1 \leq j, k \leq n$ and with $D_{k}$ for $j \neq k$. In the case $j=k$ we have $\nabla_{j} D_{j}=\nabla_{j}+D_{j} \nabla_{j}$. 
Let us define a family of linear operators $\left\{W_{j}\right\}_{j=1}^{n}$ acting on the ring $R[x]$ by

$$
W_{j}=P_{j}(D) \nabla_{j}^{m}-(-1)^{m_{j}} m^{m} \prod_{k=0}^{m-1}\left(D_{j}-k\right), j=1, \ldots, n .
$$

We claim that the family of operators $\left\{W_{j}\right\}_{j=1}^{n}$ is commutative. Indeed, using the fact that $\left\{D_{j}\right\}_{j=1}^{n}$ is a commutative family of operators and the identity $\nabla_{j}^{m} D_{j}=$ $\left(D_{j}+m\right) \nabla_{j}^{m}$ we conclude, that

$$
\begin{gathered}
W_{j} W_{k}=P_{j}(D) P_{k}\left(D_{1}, \ldots, D_{j}+m, \ldots, D_{n}\right) \nabla_{j}^{m} \nabla_{k}^{m}= \\
\left(\prod_{\mu=0}^{m_{j}+m_{k}-1}(\langle M, D\rangle+m \mu+1) \prod_{\mu=0}^{m_{j}^{\prime}+m_{k}^{\prime}-1}\left(\left\langle M^{\prime}, D\right\rangle+m \mu-1\right)\right) \nabla_{j}^{m} \nabla_{k}^{m} .
\end{gathered}
$$

Computing the composition of the operators $W_{j}$ and $W_{k}$ in the reversed order, we arrive at the same expression.

Using Lemma 2.2 and Theorem 2.4 in [14] we conclude that the $\mathcal{D}$-module associated with the modified Mellin system (2.3) is isomorphic to the quotient

$$
R[x] /\left(\sum_{j=1}^{n} W_{j} R[x]\right) .
$$

By the commutativity of the operators $W_{j}$ and Lemma 2.7 in [14, the dimension of this quotient as a complex vector space equals the product of the degrees of the operators in the Mellin system, i.e., $m^{n}$. This justifies the first claim of the theorem.

Let us now prove the second statement of the theorem. Let $I \in B$ and consider a power series of the form

$$
f_{I}(x)=\sum_{s \in m \mathbb{N}_{0}^{n}+I} \varphi(s) x^{s}
$$

where $m \mathbb{N}_{0}^{n}+I=\left\{\left(s_{1}, \ldots, s_{n}\right) \in \mathbb{N}_{0}^{n}: s_{k}=m p_{k}+i_{k}\right.$, for some $\left.p_{k} \in \mathbb{N}_{0}\right\}$. To compute the action of the operator $G_{j}$ on $f_{I}(x)$ we notice that

$$
x_{j}^{m} \frac{\partial^{m}}{\partial x_{j}^{m}}=\prod_{k=0}^{m-1}\left(\theta_{j}-k\right)
$$

and that

$$
P_{j}(\theta) f_{I}(x)=\sum_{s \in m \mathbb{N}_{0}^{n}+I} P_{j}(s) \varphi(s) x^{s} .
$$

Since $\prod_{k=0}^{m-1}\left(i_{j}-k\right)=0$ for any $I \in B$ and any $j=1, \ldots, n$, it follows, that $G_{j} f_{I}(x)=0$ if and only if

$$
\varphi(s) P_{j}(s+I)=(-1)^{m_{j}} m^{m} \varphi\left(s+m e_{j}\right) \prod_{k=0}^{m-1}\left(s_{j}+m+i_{j}-k\right) .
$$

Here $e_{j}=(0, \ldots, 1, \ldots, 0)$ with 1 in the $j$-th place. It is always possible to satisfy this relation by choosing $\varphi(s)$ in an appropriate way. Indeed, the product $\prod_{k=0}^{m-1}\left(s_{j}+\right.$ 
$\left.m+i_{j}-k\right)$ is never zero and hence the recurrent relations (2.8) can always be solved. Thus for any $I \in B$ there exists a formal series solution to (1.3) of the form (2.6). Notice that the resultant of the principal symbols of the operators in the Mellin system (1.3) is different from zero at the origin. By Proposition 2 in [12], any formal series solution to the Mellin system of the form (2.6) converges in some neighbourhood of the origin. The $m^{n}$ series $\left\{f_{I}(x)\right\}_{I \in B}$ are linearly independent since their initial monomials are different. Thus they form a convenient basis in the space of analytic solutions to the Mellin system. This completes the proof of Theorem 2.1].

Definition 2.2. We call an analytic solution $y(x)$ to the Mellin system (1.3) defined near the origin a generating solution to this system if for any $I \in B$,

$$
\partial_{I} y(0):=\frac{\partial^{|I|} y(0)}{\partial x_{1}^{i_{1}} \ldots \partial x_{n}^{i_{n}}} \neq 0 .
$$

As we will see in Theorem 2.4 a generating solution gives rise to a basis in the solution space of the Mellin system. On the other hand, the sum of the elements of a convenient basis is a generating solution to (1.3).

Example 2.3. The Mellin equation associated with the quadratic equation $y^{2}+$ $x y-1=0$ has the form

$$
\left(x^{2}+4\right) y^{\prime \prime}+x y^{\prime}-y=0 .
$$

A convenient basis in the solution space of this second-order differential equation is given by the functions $f_{1}(x)=x, f_{2}(x)=\sqrt{x^{2}+4}$. Another basis is given by the roots $y_{i}=\frac{1}{2}\left(-f_{1} \pm f_{2}\right)$ of the quadratic equation $y^{2}+x y-1=0$, which are generating solutions.

We denote by $\varepsilon$ the $m$-th primitive root of unity $\varepsilon:=e^{2 \pi i / m}$.

Theorem 2.4. Let $y(x)$ be an analytic function near the origin which is a solution to the Mellin system (1.3). Then, $y(x)$ is a generating solution if and only if the family of $m^{n}$ functions $y_{I}(x)=y\left(\varepsilon^{i_{1}} x_{1}, \ldots, \varepsilon^{i_{n}} x_{n}\right), I=\left(i_{1}, \ldots, i_{n}\right) \in B$ is a basis in the solution space of (1.3).

Proof. Assume that $y(x)$ is a generating solution. The differential operators $\theta_{k}=$ $x_{k} \frac{\partial}{\partial x_{k}}$ and $\frac{\partial^{m}}{\partial x_{k}^{m}}$ are invariant under the change of variables $t_{k}=\varepsilon^{i_{k}} x_{k}$ for any $k=$ $1, \ldots, n$. Indeed, $x_{k} \frac{\partial}{\partial x_{k}}=\varepsilon^{-i_{k}} t_{k} \frac{\partial}{\partial t_{k}} \frac{\partial t_{k}}{\partial x_{k}}=t_{k} \frac{\partial}{\partial t_{k}}$. The invariance of $\frac{\partial^{m}}{\partial x_{k}^{m}}$ follows from the identity $\varepsilon^{m}=1$. Since the differential equations in the system (1.3) only contain such operators, it follows that the function $y_{I}(x)=y\left(\varepsilon^{i_{1}} x_{1}, \ldots, \varepsilon^{i_{n}} x_{n}\right)$ solves (1.3) for any $I \in B$.

Let us show that the family of $m^{n}$ functions $\left\{y_{I}(x)\right\}_{I \in B}$ is linearly independent. This will prove the assertion of the theorem. Let

$$
y(x)=\sum_{s \in \mathbb{N}_{0}^{n}} \varphi(s) x^{s}
$$


be the Taylor series expansion of $y(x)$ around the origin. For a given $I \in B$ consider its subseries defined by

$$
f_{I}(x)=\sum_{s \in m \mathbb{N}_{0}^{n}+I} \varphi(s) x^{s},
$$

where $m \mathbb{N}_{0}^{n}+I=\left\{\left(s_{1}, \ldots, s_{n}\right) \in \mathbb{N}_{0}^{n}: s_{k}=m p_{k}+i_{k}\right.$, for some $\left.p_{k} \in \mathbb{N}_{0}\right\}$. By assumption $y(x)$ is a generating solution to (1.3). Hence the family of series $\left\{f_{I}(x)\right\}_{I \in B}$ is linearly independent since their initial monomials are different. Being subseries of a convergent power series, they all converge in some neighbourhood of the origin. It follows directly from the definition of $f_{I}(x)$ that $y(x)=\sum_{I \in B} f_{I}(x)$.

Suppose that the family of functions $\left\{y_{I}(x)\right\}_{I \in B}$ satisfies a linear relation

$$
\sum_{I \in B} c_{I} y_{I}(x) \equiv 0
$$

for some $c_{I} \in \mathbb{C}$. Let $J=\left(j_{1}, \ldots, j_{n}\right) \in B$ be a multi-index. Using the definition of the function $f_{J}$ and the identity $\varepsilon^{m}=1$ we conclude that

$$
\begin{gathered}
f_{J}\left(\varepsilon^{i_{1}} x_{1}, \ldots, \varepsilon^{i_{n}} x_{n}\right)=\sum_{s \in m \mathbb{N}_{0}^{n}+J} \varphi(s)\left(\varepsilon^{i_{1}} x_{1}\right)^{s_{1}} \ldots\left(\varepsilon^{i_{n}} x_{n}\right)^{s_{n}}= \\
\sum_{s \in m \mathbb{N}_{0}^{n}+J} \varepsilon^{i_{1} j_{1}+\ldots+i_{n} j_{n}} \varphi(s) x^{s}=\sum_{s \in m \mathbb{N}_{0}^{n}+J} \varepsilon^{\langle I, J\rangle} \varphi(s) x^{s}=\varepsilon^{\langle I, J\rangle} f_{J}(x),
\end{gathered}
$$

where $\langle I, J\rangle$ denotes the scalar product. Thus the relation (2.11) can be written in the form

$$
\begin{gathered}
0 \equiv \sum_{I \in B} c_{I} y_{I}(x)=\sum_{I \in B} c_{I} y\left(\varepsilon^{i_{1}} x_{1}, \ldots, \varepsilon^{i_{n}} x_{n}\right)= \\
\sum_{I \in B} c_{I} \sum_{J \in B} f_{J}\left(\varepsilon^{i_{1}} x_{1}, \ldots, \varepsilon^{i_{n}} x_{n}\right)=\sum_{J \in B}\left(\sum_{I \in B} c_{I} \varepsilon^{\langle I, J\rangle}\right) f_{J}(x) .
\end{gathered}
$$

The assumption that $y(x)$ is a generating solution to the Mellin system (1.3) implies that the family of functions $\left\{f_{J}\right\}_{J \in B}$ is linearly independent. It therefore follows from (2.12) that

$$
\sum_{I \in B} c_{I} \varepsilon^{\langle I, J\rangle}=0, \text { for any } J \in B
$$

The matrix of this system of linear homogeneous algebraic equations is the $n$-th exterior power of the Vandermonde matrix

$$
\left(\begin{array}{ccccc}
1 & 1 & 1 & \ldots & 1 \\
1 & \varepsilon & \varepsilon^{2} & \ldots & \varepsilon^{m-1} \\
1 & \varepsilon^{2} & \varepsilon^{4} & \ldots & \varepsilon^{2(m-1)} \\
\cdots & \cdots & \ldots & \ldots & \ldots \\
1 & \varepsilon^{m-1} & \varepsilon^{2(m-1)} & \ldots & \varepsilon^{(m-1)^{2}}
\end{array}\right)
$$

This Vandermonde matrix is nondegenerate since the numbers $1, \varepsilon, \varepsilon^{2}, \ldots, \varepsilon^{m-1}$ are all different. It is known that the eigenvalues of the exterior product of two matrices 
are products of the eigenvalues of the factors. Thus the exterior product of two nondegenerate matrices is nondegenerate and hence so is the matrix of the system (2.13). This implies that $c_{I}=0$ for any $I \in B$ and hence the functions $\left\{y_{I}(x)\right\}_{I \in B}$ are linearly independent.

Assume now that $y(x)$ is not generating and let $J \in B$ such that $\partial_{J} y(0)=0$. Then, $\partial_{J} y_{I}(0)=0$ for all $I \in B$. By Theorem 2.1 there exists a solution $f_{J}$ with $\partial_{J} f_{J}(0)=1$. Then, the family $\left\{y_{I}(x), I \in B\right\}$ cannot be a basis of solutions of the Mellin system. This completes the proof.

Recall that we denote $d=\operatorname{GCD}\left(m, m_{1}, m_{2}, \ldots, m_{n}\right)$.

Corollary 2.5. If $d>1$ then $\left\{y_{p r}\left(\varepsilon^{i_{1}} x_{1}, \ldots, \varepsilon^{i_{n}} x_{n}\right)\right\}_{I \in B}$ is a basis in the solution space to the Mellin system (1.3). Here $y_{p r}(x)$ is the principal solution (2.1) to the Mellin system. In particular, under the above assumption all solutions to the Mellin system are algebraic functions.

Proof. If $d>1$ then the term $m_{1} \nu_{1}+\ldots+m_{n} \nu_{n}-m \nu+1$ in the numerator of the coefficient of (2.1) is never zero. In particular, it is nonzero for $\left(\nu_{1}, \ldots, \nu_{n}\right) \in B$ and hence $y_{p r}(x)$ is a generating solution to the Mellin system. The conclusion of the corollary follows now from Theorem 2.4 .

Example 2.6. Consider the algebraic equation

$$
y^{6}+x_{1} y^{4}+x_{2} y^{2}-1=0 .
$$

A generating solution for the corresponding Mellin system is given by the function

$$
y\left(x_{1}, x_{2}\right)=\sqrt{-2 x_{1}+u\left(x_{1}, x_{2}\right)-\frac{12 x_{2}-4 x_{1}^{2}}{u\left(x_{1}, x_{2}\right)}},
$$

where $u\left(x_{1}, x_{2}\right)=\sqrt[3]{108+36 x_{1} x_{2}-8 x_{1}^{3}+12 \sqrt{81+54 x_{1} x_{2}-12 x_{1}^{3}+12 x_{2}^{3}-3 x_{1}^{2} x_{2}^{2}}}$. A simple computation in Maple shows that all of the initial exponents for (2.14) are present in the series expansion of $y\left(x_{1}, x_{2}\right)$, as predicted by Corollary 2.5. In contrast with Example 4.2 below, in this case it is possible to give a generating solution to the Mellin system associated with (2.14) in terms of the solutions to (2.14) itself.

Remark 2.7. From the point of view of the original algebraic equation (1.2) it is, of course, very unnatural to make the assumption that $d>1$. Indeed, the change of unknown $u(x)=y(x)^{d}$ reduces (1.2) to an equation with relatively prime exponents. However, it turns out that the Mellin system associated with this transformed equation differs essentially from the Mellin system associated with the original algebraic equation. This is illustrated by Examples 5.7 and [5.3, and by Examples 2.6] and 4.2. since in case $d>1$ all solutions are algebraic by Corollary [2.5, while as we will see, in case $d=1, n=1, m_{1}<m-1$ or $d=1, n>1$, there always exists a non-algebraic solution. Thus from the point of view of the Mellin system the case when $d>1$ cannot be trivially reduced to the case of relatively prime exponents. 


\section{A-Hypergeometric, Horn hypergeometric and Mellin systems}

Given the equation (1.1), its roots (thought of as functions of $\left.a=a_{0}, \ldots, a_{n+1}\right)$ ) satisfy the $A$-hypergeometric system with parameter $\beta:=(0,-1)$ (cf [16]), where

$$
A:=\left(\begin{array}{ccccc}
1 & 1 & \ldots & 1 & 1 \\
m & m_{1} & \ldots & m_{n} & 0
\end{array}\right) .
$$

Namely, it is the left ideal in the Weyl algebra $\mathbb{C}\left[a_{0}, \ldots, a_{n+1}, \partial_{0}, \ldots, \partial_{n+1}\right]$ generated by

(3.2) the toric operators $\partial^{u}-\partial^{v}$ for $u, v \in \mathbb{N}^{n+2}$ with $A \cdot u=A \cdot v$,

(3.3) and the Euler operators $\sum_{j=0}^{n+1} a_{j} \partial_{j}$ and $m a_{0}+\sum_{j=1}^{n} m_{j} a_{j} \partial_{j}+1$.

Note that the corresponding $A$-hypergeometric system is the same as the hypergeometric system associated with the matrix

$$
A^{\prime}:=\left(\begin{array}{ccccc}
1 & 1 & \ldots & 1 & 1 \\
m / d & m_{1} / d & \ldots & m_{n} / d & 0
\end{array}\right)
$$

and homogeneity $\beta^{\prime}=(0,-1 / d)$.

Consider now the following matrix $\mathcal{B} \in \mathbb{Z}^{(n+1) \times m}$ :

$$
\mathcal{B}:=\left(\begin{array}{cccc}
-m_{1} & -m_{2} & \ldots & -m_{n} \\
m & 0 & \ldots & 0 \\
0 & m & \ldots & 0 \\
\vdots & \vdots & \vdots & \vdots \\
0 & 0 & \ldots & m \\
-m_{1}^{\prime} & -m_{2}^{\prime} & \ldots & -m_{n}^{\prime}
\end{array}\right)
$$

where as above $m_{i}^{\prime}=m-m_{i}, i=1, \ldots, n$. Let $c=(-1 / m, 0 \ldots, 0,1 / m)$ and denote by $\operatorname{Horn}_{\mathcal{B}}(c)=\left\langle H_{1}, \ldots, H_{n}\right\rangle$ the corresponding Horn system, as in Definition 2.1 in [6], which we now recall. For $j=1, \ldots, n$, the Horn operator $H_{j}$ in the variables $w=\left(w_{1}, \ldots, w_{n}\right)$ equals

$$
\prod_{k=0}^{m-1}\left(m \theta_{j}-k\right)-w_{j} \prod_{k=0}^{m_{j}-1}\left(-m_{1} \theta_{1}-\ldots-m_{n} \theta_{n}-\frac{1}{m}-k\right) \prod_{k=0}^{m_{j}^{\prime}-1}\left(-m_{1}^{\prime} \theta_{1}-\ldots-m_{n}^{\prime} \theta_{n}+\frac{1}{m}-k\right) .
$$

We deduce from Lemma 5.1 and Corollary 5.2 in [6] the following result.

Lemma 3.1. Let $\psi\left(w_{1}, \ldots, w_{n}\right)$ be a function of $n$ complex variables and let $\varphi(a)$ be the following function of $n+2$ variables (defined in suitable simply connected open sets):

$$
\varphi(a)=\left(\frac{a_{0}}{a_{n+1}}\right)^{-\frac{1}{m}} \psi\left(\frac{a_{1}^{m}}{a_{0}^{m_{1}} a_{n+1}^{m_{1}^{\prime}}}, \ldots, \frac{a_{n}^{m}}{a_{0}^{m_{n}} a_{n+1}^{m_{n}^{\prime}}}\right) .
$$

Then, $\varphi$ is a solution to the A-hypergeometric system with parameter $\beta$ if and only if $\psi$ is a solution to $\operatorname{Horn}_{\mathcal{B}}(c)$. 
Setting $a_{0}=1, a_{n+1}=-1$, we deduce the relation

$$
\varphi\left(1, a_{1}, \ldots, a_{n},-1\right)=(-1)^{\frac{1}{m}} \psi\left((-1)^{m_{1}^{\prime}} a_{1}^{m}, \ldots,(-1)^{m_{n}^{\prime}} a_{n}^{m}\right) .
$$

We thus make the change of variables $w_{j}=(-1)^{m_{j}^{\prime}} x_{j}^{m}$ and we get that given a holomorphic function $\psi\left(w_{1}, \ldots, w_{n}\right)$ as above, the function

$$
\mu\left(x_{1}, \ldots, x_{n}\right):=\psi(w(x))=\psi\left((-1)^{m_{1}^{\prime}} x_{1}^{m}, \ldots,(-1)^{m_{n}^{\prime}} x_{n}^{m}\right)
$$

verifies

$$
(-1)^{\frac{1}{m}} \mu\left(a_{1}, \ldots, a_{n}\right)=\varphi\left(1, a_{1}, \ldots, a_{n},-1\right) .
$$

We now translate the Horn operators in the $w$ variables to the $x$ variables. Since $w_{j} \frac{\partial}{\partial w_{j}}=\frac{1}{m} x_{j} \frac{\partial}{\partial x_{j}}$, we get for all $j=1, \ldots, m$,

$$
H_{j}^{\prime}:=\prod_{k=0}^{m-1}\left(\theta_{j}-k\right)-(-1)^{m_{j}^{\prime}} x_{j}^{m} \prod_{k=0}^{m_{j}-1}\left(-\frac{m_{1}}{m} \theta_{1}-\ldots-\frac{m_{n}}{m} \theta_{n}-\frac{1}{m}-k\right) \circ
$$

$$
\prod_{k=0}^{m_{j}^{\prime}-1}\left(-\frac{m_{1}^{\prime}}{m} \theta_{1}-\ldots-\frac{m_{n}^{\prime}}{m} \theta_{n}+\frac{1}{m}-k\right)
$$

where now $\theta_{1}, \ldots, \theta_{n}$ refer to derivatives with respect to the variables $\left(x_{1}, \ldots, x_{n}\right)$, i.e., $\theta_{i}=x_{i} \frac{\partial}{\partial x_{i}}, i=1, \ldots, n$.

Note that $\prod_{k=0}^{m-1}\left(\theta_{j}-k\right)=x_{j}^{m}\left(\frac{\partial}{\partial x_{j}}\right)^{m}$. Multiplying $H_{j}^{\prime}$ by $(-1)^{m+1} m^{m}$ and dividing by $x_{j}^{m}$ we get the Mellin operators (1.3).

We immediately deduce:

Lemma 3.2. A holomorphic function $\mu\left(x_{1}, \ldots, x_{n}\right)$ is annihilated by the Mellin operators (1.3) if and only if the function $\psi\left(w_{1}, \ldots, w_{n}\right)$ verifies the Horn system (3.6), where $\mu$ and $\psi$ are related by (3.8) (in suitable open sets).

We now take advantage of these translations to provide a new proof of the rank of the Mellin system stated in Theorem 2.1. Note that the greatest common divisor $g$ of the maximal minors of $B$ equals $g=m^{n-1} d$. The normalized volume of $A$ equals $m / d$. Moreover, by the results in [7, the lattice ideal $I_{B}$ is a complete intersection. We deduce from [6, Theorem 2.5] that the rank of $\operatorname{Horn}_{\mathcal{B}}(c)$ equals $m^{n-1} d \times m / d=m^{n}$. Using the translation of solutions in Lemma 3.2. it is easy to check that the holonomic rank of the Mellin system is also $m^{n}$.

Theorem 3.3. In suitable open sets, given an A-hypergeometric function $\varphi(a)$ with parameter $\beta=(0,-1)$, the function of $n$ variables defined by specialization

$$
\mu\left(x_{1}, \ldots, x_{n}\right)=\varphi\left(1, x_{1}, \ldots, x_{n},-1\right),
$$

is a solution to the Mellin system.

Proof. Given a choice of $m$-roots of the coordinates, define

$$
\psi\left(w_{1}, \ldots, w_{n}\right):=\mu\left(\varepsilon^{-m_{1}^{\prime}} w_{1}^{\frac{1}{m}}, \ldots, \varepsilon^{-m_{n}^{\prime}} w_{n}^{\frac{1}{m}}\right),
$$


where $\varepsilon^{\prime}$ is a primitive $m$-root of -1 . Note that $w_{i}=(-1)^{m_{i}^{\prime}}\left(\varepsilon^{\prime-m_{i}^{\prime}} w_{i}^{\frac{1}{m}}\right)^{m}, i=$ $1, \ldots, n$. By Lemma 3.2, it is enough to verify that $\psi$ is a solution to the Horn system. Now, by Lemma 3.1 this happens if and only if the function $\varphi^{\prime}$ defined by:

$$
\varphi^{\prime}(a):=\left(\frac{a_{0}}{a_{n+1}}\right)^{-\frac{1}{m}} \psi\left(\frac{a_{1}^{m}}{a_{0}^{m_{1}} a_{n+1}^{m_{1}^{\prime}}}, \ldots, \frac{a_{n}^{m}}{a_{0}^{m_{n}} a_{n+1}^{m_{n}^{\prime}}}\right),
$$

satisfies the $A$-hypergeometric system with parameter $\beta$. But calling $\lambda$ an $m$-th root of $-a_{0} / a_{n+1}$ we deduce from the definitions of the functions and the homogeneity $(0,-1)$ satisfied by $\varphi$ that

$$
\begin{aligned}
\varepsilon^{\prime} \varphi^{\prime}(a) & =\varepsilon^{\prime}\left(\frac{a_{0}}{a_{n+1}}\right)^{-\frac{1}{m}} \psi\left(a_{1}^{m} a_{n+1}^{m_{1}-m} a_{0}^{-m_{1}}, \ldots, a_{n}^{m} a_{n+1}^{m_{n}-m} a_{0}^{-m_{n}}\right) \\
& =\lambda^{-1} \mu\left(\left(-\frac{a_{1}}{a_{n+1}}\right) \lambda^{-m_{1}}, \ldots,\left(-\frac{a_{n}}{a_{n+1}}\right) \lambda^{-m_{n}}\right) \\
& =\lambda^{-1} \varphi\left(1,-\frac{a_{1}}{a_{n+1}} \lambda^{-m_{1}}, \ldots,-\frac{a_{n}}{a_{n+1}} \lambda^{-m_{n}},-1\right)=\varphi(a),
\end{aligned}
$$

and we are done.

\section{Solutions in terms of ROOTS}

Mellin not only observed in [10] that the roots $y_{1}(x), \ldots, y_{m}(x)$ of the algebraic equation (1.2)

$$
y^{m}+x_{1} y^{m_{1}}+\ldots+x_{n} y^{m_{n}}-1=0
$$

satisfy the Mellin system (1.3), but he also made the following easy observation. Given the principal solution $y_{p r}(x)$ in (2.1), all the solutions have the form

$$
\eta y_{p r}\left(\eta^{m_{1}} x_{1}, \ldots, \eta^{m_{n}} x_{n}\right)
$$

where $\eta$ runs through the $m$-roots of 1 .

It is also clear that for any choice of $I=\left(i_{1}, \ldots, i_{n}\right) \in \mathbb{N}^{n}$, the function $y_{I}(x)=$ $y_{p r}\left(\varepsilon^{i_{1}} x_{1}, \ldots, \varepsilon^{i_{n}} x_{n}\right)$ is a root of the algebraic equation

$$
y^{m}+\varepsilon^{i_{1}} x_{1} y^{m_{1}}+\ldots+\varepsilon^{i_{n}} x_{n} y^{m_{n}}-1=0,
$$

where, as in Section 2, we denote $\varepsilon=e^{2 \pi i / m}$. Moreover, as we have already remarked, all these functions lie in the solution space of (1.3). So, the Mellin system has not only the roots of the algebraic equation (1.2) as solutions, but also the roots of the associated equations $(I)$. It is clear that we only get $m^{n}$ different equations this way, and we could parametrize them taking $I \in B$. We denote by $Y$ the $\mathbb{C}$-vector space generated by all the roots of all the equations of the form $(I)$. The roots of the original equation (1.2) correspond precisely to the functions $\varepsilon^{j} y_{\left(j m_{1}, \ldots, j m_{n}\right)}, j=$ $0, \ldots, m-1$. Note that these $m$ functions are all distinct, but of course they are not linearly independent if $d>1$ or if $d=1$ and $m_{1}<m-1$. In case $d>1$ we have seen in Corollary 2.5 that $Y$ coincides with the solutions space of the Mellin system.

Example 4.1. The polyquadratic equation. Consider the equation

$$
y^{2 k}+x y^{k}-1=0 .
$$


Its $2 k$ roots are $\varepsilon^{j} \sqrt[k]{\left(-x \pm \sqrt{x^{2}+4}\right) / 2}, j=0, \ldots, k-1$, where $\varepsilon$ is the primitive $k$-th root of unity. Observe that these roots span a vector space of dimension 2. A basis in the $2 k$-dimensional solution space of the Mellin equation associated with (4.1) is given by the functions $\sqrt[k]{-x \pm \sqrt{x^{2}+4 \varepsilon^{j}}}, j=0, \ldots, k-1$.

For the rest of this section we will then assume that $d=1$. Our task is to explain and generalize the following example.

Example 4.2. The general cubic. Consider the algebraic equation

$$
y^{3}+x_{1} y^{2}+x_{2} y-1=0 .
$$

The corresponding Mellin system is given by

$$
\begin{aligned}
27 \theta_{1}\left(\theta_{1}-1\right)\left(\theta_{1}-2\right) y & =x_{1}^{3}\left(2 \theta_{1}+\theta_{2}+1\right)\left(2 \theta_{1}+\theta_{2}+4\right)\left(\theta_{1}+2 \theta_{2}-1\right) y \\
-27 \theta_{2}\left(\theta_{2}-1\right)\left(\theta_{2}-2\right) y & =x_{2}^{3}\left(2 \theta_{1}+\theta_{2}+1\right)\left(\theta_{1}+2 \theta_{2}-1\right)\left(\theta_{1}+2 \theta_{2}+2\right) y
\end{aligned}
$$

The three solutions to (4.2) are linear combinations of the following functions:

$$
y_{1}\left(x_{1}, x_{2}\right)=x_{1}, \quad y_{2}\left(x_{1}, x_{2}\right)=\sqrt[3]{u\left(x_{1}, x_{2}\right)}, \quad y_{3}\left(x_{1}, x_{2}\right)=\frac{3 x_{2}-x_{1}^{2}}{\sqrt[3]{u\left(x_{1}, x_{2}\right)}},
$$

where $u\left(x_{1}, x_{2}\right)=108+36 x_{1} x_{2}-8 x_{1}^{3}+12 \sqrt{81+54 x_{1} x_{2}-12 x_{1}^{3}+12 x_{2}^{3}-3 x_{1}^{2} x_{2}^{2}}$. The expansions of $y_{2}$ and $y_{3}$ into power series around the origin are given by

$$
y_{2}=6+\frac{2}{3} x_{1} x_{2}-\frac{4}{27} x_{1}^{3}+\frac{2}{27} x_{2}^{3}-\frac{4}{27} x_{1}^{2} x_{2}^{2}+\ldots, \quad y_{3}=\frac{1}{2} x_{2}-\frac{1}{6} x_{1}^{2}-\frac{1}{18} x_{1} x_{2}^{2}+\ldots
$$

In this example, the set of initial exponents is given by $B=\left\{(i, j) \in \mathbb{Z}^{2}: 0 \leq i, j \leq\right.$ $2\}$. The expansions of $y_{1}, y_{2}$, and $y_{3}$ into power series contain 7 of the 9 elements in $B$. This could be also checked differentiating (4.2) implicitly and evaluating at 0 . The 2 missing exponents are $(2,1)$ and $(0,2)$. Thus the solution space of (4.3) contains a two-dimensional subspace which is not spanned by solutions to (4.2).

Remark that the roots of the equations $(I)$ above with any index set of the form $I_{j}=\left(j m_{1}, \ldots, j m_{n}\right)$ for any $j=1, \ldots, m-1$, are just constant multiples of the roots of the original equation (1.2) (corresponding to $j=0$ ). More generally, there are $m^{n-1}$ subsets $G^{(1)}, \ldots, G^{\left(m^{n-1}\right)}$ of algebraic equations of the form $(I)$, each of them consisting of $m$ equations, such that for any pair of equations $(I),(J)$ in the same subset $G^{(k)}$, the corresponding solutions only differ by multiplicative constants. Denote by $\Gamma=\left\{I^{(1)}, \ldots, I^{\left(m^{n-1}\right)}\right\}$, where each $I^{(k)} \in B$ is a choice of an index set in the subset $G^{(k)}$ of algebraic equations, for all $k=1, \ldots, m^{n-1}$. The analytic roots of the algebraic equation $\left(I^{(k)}\right)$ defined in a small neighborhood of the origin will be denoted by $y_{1}^{(k)}, \ldots, y_{m}^{(k)}$.

Given a complex vector $c=\left(c^{(1)}, \ldots, c^{\left(m^{n-1}\right)}\right)$, denote by $\chi_{c}$ the function defined by

$$
\chi_{c}=\sum_{k=1}^{m^{n-1}} c^{(k)}\left(y_{1}^{(k)} \log \left(y_{1}^{(k)}\right)+\cdots+y_{m}^{(k)} \log \left(y_{m}^{(k)}\right)\right)
$$


where log denotes a holomorphic branch of the logarithm defined in the union of the images of the roots.

When the exponents $m, m_{1}, \ldots, m_{n}$ are relatively prime, is it shown in 4, Th. 2.4] (see also [9, Cor. 3.6]) that the dimension of the vector space generated by the solutions to the algebraic equation (1.2) is equal to $m$ if $m_{1}=m-1$ and it is equal to $m-1$ if $m_{1}<m-1$. Thus, when $d=1$ the only possible linear relation between the solutions to the algebraic equation (1.2) is the obvious one: the roots of (1.2) sum up to zero if the coefficient by $y^{m-1}$ in (1.2) is zero.

Our main result is the following.

Theorem 4.3. The space $Y$ coincides with the space of algebraic solutions to the Mellin system of equations around the origin. Its dimension equals $\operatorname{dim} Y=m^{n}-$ $m^{n-1}+1$ in case $m_{1}=m-1$ and $\operatorname{dim} Y=m^{n}-m^{n-1}$ otherwise. The space of relations

$$
R:=\left\{c \in \mathbb{C}^{m^{n-1}} / \sum_{k=1}^{m^{n-1}} c^{(k)}\left(y_{1}^{(k)}+\cdots+y_{m}^{(k)}\right)=0\right\}
$$

has dimension $\operatorname{dim} R=m^{n-1}-1$ in case $m_{1}=m-1$ and $\operatorname{dim} R=m^{n-1}$ otherwise. Moreover, for any nonzero $c \in R$, the function $\chi_{c}$ defined by (4.4) is a non-algebraic solution to the Mellin system, the space

$$
S:=\left\{\chi_{c} / c \in R\right\}
$$

has dimension $\operatorname{dim} S=\operatorname{dim} R$ and the sum $S \oplus Y$ is direct and equals the full solution space of the Mellin system.

When $n=1, S$ is nonzero only when $m_{1}<m-1$ and in this case it is spanned by the non-algebraic function $\chi=y_{1} \log \left(y_{1}\right)+\cdots+y_{m} \log \left(y_{m}\right)$, where $y_{1}(x), \ldots, y_{m}(x)$ denote the roots of $y^{m}+x y^{m_{1}}-1=0$, while the space of algebraic solutions $Y$ has dimension $m-1$. This is the content of Theorem 3.5 in [4] for $A$-hypergeometric systems associated to monomial curves, which can be directly translated to the Mellin equation via our computations in Section 3. So, Theorem 4.3 is inspired by and generalizes this result. When translating the Mellin system to the homogeneous setting as in Section 3, the ocurrence of logarithmic solutions is explained by the results in 5 .

Example 4.4. (Example 4.2 continued) In this case $\operatorname{dim} R=\operatorname{dim} S=3-1=2$, as we have seen. There are 3 subsets of 3 equations each and we could choose the following representatives to form our set $\Gamma$ :

$$
\begin{aligned}
& y^{3}+x_{1} y^{2}+x_{2} y-1=0 \\
& y^{3}+x_{1} y^{2}+\varepsilon x_{2} y-1=0, \\
& y^{3}+x_{1} y^{2}+\varepsilon^{2} x_{2} y-1=0,
\end{aligned}
$$

where $\varepsilon$ is a primitive cubic root of 1 , and $I_{1}=(0,0), I_{2}=(0,1), I_{3}=(0,2)$.

A basis of relations in $R$ is given for instance by the two relations $(1,-1,0)$, $(1,0,-1)$, since the sum of the roots of each of the 3 equations above equals the same value $x_{1}$. On the other side, the space $Y$ spanned by the functions $y_{j}^{(k)}, j, k=1,2,3$ 
has dimension 7 . So a basis of the solutions near the origin is given by choosing seven of these roots together with the two non-algebraic functions $\chi_{(1,-1,0)}, \chi_{(1,0,-1)}$.

Proof of Theorem 4.3. We begin by computing the dimension of $Y$. Call $\varphi_{\nu}$, for $\nu \in \mathbb{N}_{0}^{n}$, the coefficients in the expansion (2.1) of the principal solution $y=y_{p r}$ of the algebraic equation (1.2):

$$
\varphi_{\nu}=\frac{(-1)^{|\nu|}}{m^{|\nu|}} \frac{\prod_{\mu=1}^{|\nu|-1}\left(m_{1} \nu_{1}+\ldots+m_{n} \nu_{n}-m \mu+1\right)}{\nu_{1} ! \ldots \nu_{n} !} .
$$

Note that when $d>1$, the solution $y_{p r}$ is generating. This is proven in Theorem 2.4 whose proof we now generalize with similar notations.

Let $f_{I}, I \in B$, be defined as in (2.10). Observe that when $\varphi_{I}=0$, then $\varphi_{I^{\prime}}=0$ for all $I^{\prime} \in I+m \mathbb{N}_{0}^{n}$. It follows that when $\varphi_{I}=0$ then $f_{I}=0$. Let $B^{\prime}=\{I \in$ $\left.B: \varphi_{I} \neq 0\right\}$. We claim that $\operatorname{dim} Y=\# B^{\prime}$. Clearly, $y=\sum_{I \in B^{\prime}} f_{I}$ and the family $\left\{f_{I}: I \in B^{\prime}\right\}$ is linearly independent. Suppose that the family of functions $\left\{y_{I}\right\}_{I \in B}$ satisfies a linear relation

$$
\sum_{I \in B} c_{I} y_{I}(x) \equiv 0
$$

for some $c_{I} \in \mathbb{C}$. Then,

$$
\sum_{J \in B^{\prime}}\left(\sum_{I \in B} c_{I} \varepsilon^{\langle I, J\rangle}\right) f_{J}(x)=0
$$

which implies that

$$
\sum_{I \in B} c_{I} \varepsilon^{\langle I, J\rangle}=0, \text { for any } J \in B^{\prime} .
$$

In fact, $\left(c_{I}\right)_{I \in B}$ satisfy (4.6) if and only if these last equalities hold. Since the full matrix $\left(\varepsilon^{\langle I, J\rangle}\right)_{I, J \in B}$ is of maximal rank, it follows that the system of equations (4.7) has rank $\# B^{\prime}$ and so the dimension of the space of linear relations $R^{\prime}$ among the algebraic functions $\left\{y_{I}, I \in B\right\}$ is equal to $\# B-\# B^{\prime}$. Dualizing, we conclude that their linear span $Y$ has dimension $\# B^{\prime}$, as wanted.

We now prove that the cardinality of $B^{\prime}$ equals $m^{n}-m^{n-1}+1$ in case $m_{1}=m-1$ and $\operatorname{dim} Y=m^{n}-m^{n-1}$ otherwise. Call $B^{\prime \prime}=B \backslash B^{\prime}$ its complement in $B$. It is clear that $\nu \in B^{\prime \prime}$ if and only if there exists a positive integer $\mu$ strictly smaller than $|\nu|$ such that

$$
m_{1} \nu_{1}+\cdots+m_{n} \nu_{n}-m \mu+1=0
$$

which we simply write as $\langle M, \nu\rangle-m \mu+1=0$, where $M=\left(m_{1}, \ldots, m_{n}\right)$. Note that when this equality holds, it follows that the integer $\mu$ satisfies $1 \leq \mu \leq|\nu|$, since $0<m_{n}<\cdots<m_{1}<m$ and all $\nu_{i}$ are non-negative. Moreover, it is easy to check that the equality $\mu=|\nu|$ can only hold when $m_{1}=m-1$ and $\mu=\nu_{1}=1$ and $\nu_{j}=0$ for any $j>1$. So it is enough to prove that the cardinality of the set

$$
\{\nu \in B:\langle M, \nu\rangle=-1 \bmod (m)\}
$$

equals $m^{n-1}$. We claim that for any $r=0, \ldots, m-1$ there exist precisely $m^{n-1}=$ $m^{n} / m$ solutions $\nu \in B$ to the modular equation $\langle M, \nu\rangle=r \bmod (m)$. Since we 
are assuming that $d=1$, there exists an integer $n$-tuple $X=\left(x_{1}, \ldots, x_{n}\right)$ satisfying $\langle M, X\rangle=1 \bmod (m)$ and so the equation $\langle M, \nu\rangle=r \bmod (m)$ has a solution for any $r$. Then it is easy to see that for any $\nu,\{\langle M, \nu\rangle \bmod (m),\langle M, \nu+X\rangle$ $\bmod (m), \ldots,\langle M, \nu+(m-1) X\rangle \bmod (m)\}$ takes all the values $r=0, \ldots, m-1$ precisely once. Moreover, two such subsets are disjoint or equal, from which the result follows since the cardinality of $B$ is $m^{n}$.

It follows that the space $R^{\prime}$ of linear relations among the algebraic functions $\left\{y_{I}, I \in B\right\}$ has dimension $m^{n-1}-1$ in case $m_{1}=m-1$ and $m^{n-1}$ otherwise. The relations $R$ define in principle a subspace of $R^{\prime}$ corresponding to those vectors $c$ which have a special pattern of repeated coordinates, but it is easy to see that it has the same dimension. Indeed it is very easy to find a linear relation between the sum of the roots in each equation (which equals minus the coefficient of $y^{m-1}$, which is a constant multiple of $x_{1}$ if $m_{1}=m-1$ and 0 otherwise), with the sum of the roots of the original equation (1.2). This gives $m^{n-1}-1$ independent linear relations in $R$ and we get moveover the relation $c=(1,0, \ldots, 0)$ in case $m_{1}<m-1$. We can explicitly list a basis of the space $R$ : the vectors $\left\{e_{1}-e_{2}, \ldots, e_{1}-e_{m^{n-1}}\right\}$ when $m_{1}=m-1$ and the vectors $\left\{e_{1}, \ldots, e_{m^{n-1}}\right\}$ otherwise.

Then, $R=R^{\prime}$ and we recover in particular the quoted result in [4, Th. 2.4] about the only possible linear relation among the roots of a single sparse algebraic equation with generic coefficients.

In order to see that for any $c \in R$ the function $\chi_{c}$ is a solution to the Mellin system, we will need the translations described in Section 3 between the Mellin system and the corresponding Horn and $A$-hypergeometric systems. This will allow us to use the results in [4] based on the study of the relation between lattice hypergeometric ideals and $A$-hypergeometric ideals explained in [6]. We sketch the main ingredients of the proof and refer the reader to [4, 6] for the details. Since the lattice generated by the columns of the matrix $\mathcal{B} \in \mathbb{Z}^{(n+1) \times m}$ in (3.5) equals $m^{n-1}$ and the associated lattice ideal is a complete intersection, the Horn system (3.6) gets translated by homogenization (as in Lemma 3.1) to the hypergeometric system $\mathcal{H}_{\mathcal{B}}$ corresponding to this lattice. We get $m^{n-1}$ hypergeometric systems $\mathcal{H}_{i}, i=0, \ldots, m^{n-1}-1$ which are torus translates of the $A$-hypergeometric system, all with the same homogeneity $\beta=(0,-1)$ and such that all solutions of any $\mathcal{H}_{i}$ satisfy the system $\mathcal{H}_{\mathcal{B}}$. The different functions $y_{I} \in Y$ which satisfy the Mellin system, and thus the Horn system after the change of variables in Lemma 3.2, satisfy (after $A$-homogenization as in Lemma 3.1) different systems in the family $\mathcal{H}_{i}, i=0, \ldots, m^{n-1}-1$ according to $(I)$. Each system is satisfied precisely by $m$ of these homogenized functions, which we denote by $z_{I}(a)$. The functions $z_{I}(a)$ also satisfy the corresponding algebraic equation:

$$
a_{m} z^{m}+\varepsilon^{i_{1}} a_{1} z^{m_{1}}+\varepsilon^{i_{2}} a_{2} z^{m_{2}}+\ldots+\varepsilon^{i_{n}} a_{n} z^{m_{n}}+a_{n+1}=0 .
$$

In particular, when $I=\left(i_{1}, \ldots, i_{n}\right)$ with $i_{k}=j m_{k} \bmod (m)$ for all $k=1, \ldots, n$ and some fixed $j=0, \ldots, m-1$, the function $z_{I}$ satisfies the differential $A$-hypergeometric system recalled in Section 3 and the algebraic equation (1.1). Recall that $y_{I}(x)=$ $z_{I}\left(1, x_{1}, \ldots, x_{n},-1\right)$ as in Theorem 3.3. Changing a little bit the notation of the homogeneous roots as in (4.4), in order to prove that the function $\chi_{c}$ is a solution 
of the Mellin system, it is enough to prove that the homogenized function

$$
\bar{\chi}_{c}:=\sum_{k=1}^{m^{n-1}} c^{(k)}\left(z_{1}^{(k)} \log \left(z_{1}^{(k)}\right)+\cdots+z_{m}^{(k)} \log \left(z_{m}^{(k)}\right)\right)
$$

satisfies the lattice hypergeometric system $\mathcal{H}_{\mathcal{B}}$. Then, it is possible to prove that each of the functions

$$
\alpha^{(k)}:=z_{1}^{(k)} \log \left(z_{1}^{(k)}\right)+\cdots+z_{m}^{(k)} \log \left(z_{m}^{(k)}\right)
$$

is annihilated by the toric operators in the corresponding torus translate of the $A$ hypergeometric system as in the proof of Proposition 3.2 in 4]. Moreover, the fact that $c$ lies in $R$ allows one to prove that the whole sum $\bar{\chi}_{c}$ satisfies the homogeneity equations.

The theorem will be proved if we show the following statement: If a linear combination $\chi_{\lambda}:=\sum_{i=1}^{s} \lambda_{i} \chi_{c[i]}$ is algebraic, where $\lambda_{i} \in \mathbb{C}$ and $c[1], \ldots, c[s] \in R$, then $\sum_{i=1}^{s} \lambda_{i} c[i]=0$. Note that this implies in particular (since the function 0 is algebraic) that the dimension of the linear span of the functions $\left\{\chi_{c}: c \in R\right\}$ equals the dimension of $R$, from which we deduce not only that the sum $S \oplus Y$ is direct but also that it equals the full solution space of the Mellin system.

In order to prove the above statement, we observe that if $\chi_{\lambda}$ is an algebraic function of $\left(x_{1}, \ldots, x_{n}\right)$ then, the homogenized function

$$
\bar{\chi}_{\lambda}:=\sum_{i=1}^{s} \lambda_{i} \sum_{k=1}^{m^{n-1}} c[i]^{(k)} \alpha^{(k)}
$$

is an algebraic function of $a=\left(a_{m}, a_{m_{1}}, \ldots, a_{m_{n}}, a_{n+1}\right)$. But now, as in [4, Th. 3.5], we fix a point $a$ with $\left|a_{n+1}\right|$ sufficiently small relative to the absolute values of the other coordinates and consider the loop $\gamma$ defined by

$$
\gamma(\theta):=\left(a_{m}, a_{m_{1}}, \ldots, a_{m_{n}}, \exp \left(2 \pi i m_{n} \theta\right) a_{n+1}\right) .
$$

Analytic continuation around $\gamma$ will return all the local roots in a suitable neighborhood of $a$ to their original values, while there is a subset $S^{(k)}$ containing $m_{n}<m$ of the roots, such that the logarithms of the roots in $S^{(k)}$ will be increased by $2 \pi i$. Then $\gamma^{*}\left(\alpha^{(k)}\right)-\alpha^{(k)}$ equals $2 \pi i$ times the sum of the $m_{n}$ roots in $S^{(k)}$, which cannot be zero since $R=R^{\prime}$. We denote this sum of roots by $z_{S^{(k)}}$. Therefore

$$
\gamma^{*}\left(\bar{\chi}_{\lambda}\right)-\bar{\chi}_{\lambda}=\gamma^{*}\left(\sum_{i=1}^{s} \lambda_{i} \sum_{k=1}^{m^{n-1}} c[i]^{(k)} \alpha^{(k)}\right)-\sum_{i=1}^{s} \lambda_{i} \sum_{k=1}^{m^{n-1}} c[i]^{(k)} \alpha^{(k)},
$$

which, after dividing by $2 \pi i$, equals

$$
\sum_{i=1}^{s} \lambda_{i} \sum_{k=1}^{m^{n-1}} c[i]^{(k)} z_{S^{(k)}}=\sum_{k=1}^{m^{n-1}}\left(\sum_{i=1}^{s} \lambda_{i} c[i]^{(k)}\right) z_{S^{(k)}} .
$$

Since $z_{S}^{(k)}$ is not the complete sum of all the roots corresponding to one equation, the only way in which a finite iterate of the action of $\gamma$ returns $\bar{\chi}_{\lambda}$ to its original value is when $\sum_{i=1}^{s} \lambda_{i} c[i]^{(k)}=0$ for all $k$, i.e., $\sum_{i=1}^{s} \lambda_{i} c[i]=0$, as asserted. 
We also deduce:

Corollary 4.5. The monodromy representation of the Mellin system (1.3) is always reducible.

Proof. Suppose that $n>1$. By Theorem 2.1 the dimension of the space of analytic solutions to (1.3) equals $m^{n}$. Since the solutions to the algebraic equation (1.2) span a linear space of dimension at most $m$, there is a nontrivial invariant subspace in the solution space of (1.3). If $n=1$ and $m_{1}<m-1$, then the solutions of the algebraic equation form a nontrivial invariant subspace of dimension $m-1$. Finally, for $n=1$ and $m_{1}=m-1$, the one-dimensional linear space spanned by the rational solution $x_{1}$ is invariant under the action of the monodromy.

\section{The CASE OF DIMENSION ONE}

In this section we further investigate the special case of the Mellin system of equations associated with the trinomial algebraic equation

$$
y^{m}+x y^{m_{1}}-1=0 .
$$

In this case the Mellin system (1.3) consists of a single ordinary differential equation of order $m$, namely the equation

$$
\left(\prod_{k=0}^{m_{1}-1}\left(m_{1} \theta+m k+1\right) \prod_{k=0}^{m-m_{1}-1}\left(\left(m-m_{1}\right) \theta+m k-1\right)-(-1)^{m_{1}} m^{m} \frac{d^{m}}{d x^{m}}\right) y(x)=0,
$$

where $\theta=x \frac{d}{d x}$. Let $m=d a, m_{1}=d b$, where $d=\operatorname{GCD}\left(m, m_{1}\right)$. The discriminant of the algebraic equation (5.1) (computed with respect to $y$ ) is given by $x^{a} b^{b}(a-$ $b)^{a-b}-(-1)^{b} a^{a}$. For $d=1$, this expression coincides with the leading coefficient in the differential equation (5.2). Throughout this section, we will call the differential operator in the left-hand side of (5.2) the Mellin operator and denote it by $M\left(m, m_{1}\right)$. Special instances of this operator are considered in Examples 2.35.15.3, and 4.1.

Example 5.1. Consider the cubic equation

$$
y^{3}+x y^{2}-1=0 .
$$

The corresponding Mellin equation can be written in the form

$$
\left(4 x^{3}-27\right) y^{\prime \prime \prime}+18 x^{2} y^{\prime \prime}+4 x y^{\prime}-4 y=0 .
$$

A basis in the solution space of the differential equation (5.4) is given by the functions

$$
y_{1}(x)=x, \quad y_{2}(x)=\sqrt[3]{108-8 x^{3}+12 \sqrt{81-12 x^{3}}}, \quad y_{3}(x)=x^{2} / y_{2}(x),
$$

or by the roots of the algebraic equation (5.3).

Notice that after multiplication with $x^{2}$ the differential operator defining the equation (5.4) can be factorized as follows:

$$
x^{2}\left(\left(4 x^{3}-27\right) \frac{d^{3}}{d x^{3}}+18 x^{2} \frac{d^{2}}{d x^{2}}+4 x \frac{d}{d x}-4\right)=
$$




$$
\left(\left(4 x^{4}-27 x\right) \frac{d^{2}}{d x^{2}}+\left(14 x^{3}+27\right) \frac{d}{d x}+4 x^{2}\right)\left(x \frac{d}{d x}-1\right) .
$$

This factorization makes the rational solution $y_{1}(x)=x$ obvious.

More in general, assume $m_{1}=m-1$. Since the roots $y_{1}(x), \ldots, y_{m}(x)$ of the trinomial satisfy the equation (5.2) and sum up to $x$, it follows that the operator $\theta-1$ (which is the annihilator of $x$ ) can be factored out of $M(m, m-1)$. This factorization is described in the following proposition.

Proposition 5.2. For $m_{1}=m-1$, the only nontrivial decomposition of the Mellin operator defining the equation (5.2) in the Weyl algebra of linear differential operators with polynomial coefficients is given by

$$
x^{m} M(m, 1)=\left(x^{m} \prod_{k=0}^{m-2}((m-1) \theta+m k+1)+(-m)^{m} \theta \prod_{k=2}^{m-1}(\theta-k)\right)(\theta-1) .
$$

The proof of this proposition is analogous to the proof of Proposition 5.4 below. Assume now that $m>m_{1}+1$ and $d=\operatorname{GCD}\left(m, m_{1}\right)=1$.

Example 5.3. Consider the cubic equation

$$
y^{3}+x y-1=0 .
$$

The corresponding Mellin equation can be written in the form

$$
\left(4 x^{3}+27\right) y^{\prime \prime \prime}+18 x^{2} y^{\prime \prime}+10 x y^{\prime}-2 y=0 .
$$

Since the coefficient by $y^{2}$ in (5.6) is zero, the solutions to (5.6) generate a twodimensional vector space and thus there exists a solution $\chi$ to the differential equation (5.7) which is not a solution to the algebraic equation (5.6). The following functions give a basis of solutions to the Mellin equation (5.7):

$$
\begin{gathered}
z_{1}(x)=\sqrt[3]{108+12 \sqrt{12 x^{3}+81}}, \quad z_{2}(x)=x / z_{1}(x), \\
\chi(x)=y_{1} \log \left(y_{1}\right)+y_{2} \log \left(y_{2}\right)+y_{3} \log \left(y_{3}\right)
\end{gathered}
$$

where $y_{1}(x), y_{2}(x), y_{3}(x)$ are the three (linearly dependent) solutions to (5.6).

Notice that the differential operator defining the equation (5.7) can be factorized as follows:

$$
\left(4 x^{3}+27\right) \frac{d^{3}}{d x^{3}}+18 x^{2} \frac{d^{2}}{d x^{2}}+10 x \frac{d}{d x}-2=\frac{d}{d x}\left(\left(4 x^{3}+27\right) \frac{d^{2}}{d x^{2}}+6 x^{2} \frac{d}{d x}-2 x\right) .
$$

The functions $z_{1}, z_{2}$ give a fundamental system of solutions to the second-order factor in this decomposition. This operator is described in [9].

It turns out, that the Mellin operator corresponding to $m_{1}=1$ allows a similar decomposition. 
Proposition 5.4. For $m_{1}=1$, the only nontrivial decomposition of the Mellin operator defining the equation (5.2) in the Weyl algebra of linear differential operators with polynomial coefficients is given by

$$
M(m, 1)=\frac{d}{d x}\left(x \prod_{k=0}^{m-2}((m-1) \theta+m k-1)+m^{m} \frac{d^{m-1}}{d x^{m-1}}\right) .
$$

Proof. The validity of (5.8) follows from the Weyl algebra identity $\theta+1=\frac{d}{d x} \circ$ $x$. Here ' $O$ ' denotes the composition of differential operators in the Weyl algebra. Let us denote the second factor in the right-hand side of (5.8) by $M_{\text {alg. }}$. We would like to show that the differential equation $M_{\text {alg }} y=0$ has irreducible monodromy. Multiplying this equation with $x^{m-1}$, using the identity (2.7) and making the change of variables $t=x^{m}$, we arrive at the equation

$$
\left((m-1)^{m-1} t \prod_{i=0}^{m-2}\left(\theta_{t}+\frac{m i-1}{m(m-1)}\right)+m^{m} \prod_{j=0}^{m-2}\left(\theta_{t}-\frac{j}{m}\right)\right) y=0,
$$

where $\theta_{t}=t \frac{d}{d t}$. By Proposition 3.3 in [2] the monodromy of the differential equation (5.9) is reducible if and only if $(m i-1) / m(m-1)+j / m \in \mathbb{Z}$, for some $i, j=$ $0, \ldots, m-2$. This condition can only be satisfied when $(m i-1) / m(m-1)+j / m=1$. Clearing the denominators and considering the obtained relation modulo $m$ we conclude that it can never be satisfied. Thus the monodromy of (5.9) is irreducible and hence $M_{\text {alg }}$ does not admit any further decomposition.

In case $d>1$ the Mellin operator always factors.

Proposition 5.5. There exist d linear operators $Q_{0}, \ldots, Q_{d-1}$ of order $\frac{m}{d}$ with coefficients in $\mathbb{C}(x)$ such that $M\left(m, m_{1}\right)=Q_{0} \ldots Q_{d-1}$.

Proof. According to Corollary 2.5] and the discussion in Section 4, all solutions to $M\left(m, m_{1}\right)$ are algebraic (so its associated Galois group coincides with the monodromy group) and a basis of solutions is given by the functions

$$
\left\{f_{j, k}(x):=\varepsilon^{j} y_{p r}\left(\varepsilon^{j m_{1}+k} x\right), j=0, \ldots, m / d-1, k=0, \ldots, d-1\right\},
$$

where $\varepsilon$ is a primitive $m$-root of 1 and $y_{p r}$ is the principal solution described in (2.1). For each $k=0, \ldots, d-1$, the $m / d$ functions $\left\{f_{j, k}(x), j=0, \ldots, m_{d-1}\right\}$ are the (linearly independent) roots of the algebraic equation

$$
y^{m}+\varepsilon^{k} x y^{m_{1}}-1=0, \quad k=0, \ldots, d-1 .
$$

Then, they span a monodromy invariant subspace $V_{k}$ of the solutions $V$ to $M\left(m, m_{1}\right)$ of dimension $m / d$, which gives a right factor $L_{k}$ of order $m / d$ of the Mellin operator whose solution space equals $V_{k}$. Moreover, we have the direct sum decomposition $V=V_{0} \oplus \cdots \oplus V_{d-1}$. Then, it follows by the results in [15 that there exist operators $Q_{0}, \ldots, Q_{d-1}$ (with $Q_{i}$ equivalent to $L_{i}$ in the sense of [15]) such that $M\left(m, m_{1}\right)$ decomposes as $M\left(m, m_{1}\right)=Q_{0} \ldots Q_{d-1}$. 
Example 5.6. Let $n=1, m=4, m_{1}=2$. The Mellin equation associated with the algebraic equation $y^{4}+x y^{2}-1=0$ is given by

$$
\left(16 x^{4}-256\right) y^{(4)}+160 x^{3} y^{\prime \prime \prime}+360 x^{2} y^{\prime \prime}+120 x y^{\prime}-15 y=0 .
$$

In accordance with Corollary 2.5, a basis in the solution space of (5.10) is given by solutions to the algebraic equations $y^{4}+x y^{2}-1=0$ and $y^{4}+i x y^{2}-1=0$. After a suitable normalization this basis is given by the functions $\sqrt{-x \pm \sqrt{x^{2} \pm 4}}$. The solution space of (5.10) splits into two 2-dimensional invariant subspaces spanned by $\sqrt{-x \pm \sqrt{x^{2}+4}}$ and $\sqrt{-x \pm \sqrt{x^{2}-4}}$. This decomposition of the solution space corresponds to the factorization of the differential operator defining (5.10):

$$
\begin{aligned}
& \left(16 x^{4}-256\right) \frac{d^{4}}{d x^{4}}+160 x^{3} \frac{d^{3}}{d x^{3}}+360 x^{2} \frac{d^{2}}{d x^{2}}+120 x \frac{d}{d x}-15= \\
& \left(\left(4 x^{2}-16\right) \frac{d^{2}}{d x^{2}}+20 x \frac{d}{d x}+15\right)\left(\left(4 x^{2}+16\right) \frac{d^{2}}{d x^{2}}+4 x \frac{d}{d x}-1\right) .
\end{aligned}
$$

Example 5.7. Let $n=1, m=6, m_{1}=2$ and consider the algebraic equation

$$
y^{6}+x y^{2}-1=0
$$

The Mellin equation associated with (5.11) is given by

$$
\left(1024 x^{6}-46656\right) y^{(6)}+27648 x^{5} y^{(5)}+242816 x^{4} y^{(4)}+
$$

$$
818944 x^{3} y^{\prime \prime \prime}+955780 x^{2} y^{\prime \prime}+236180 x y^{\prime}-6545 y=0 .
$$

A basis in the space of solutions to (5.12) is given by $\left\{f\left(\varepsilon^{i} x\right), i=0, \ldots, 5\right\}$, where $\varepsilon=e^{\pi i / 3}$ and $f(x)$ is the principal solution to (5.11). The differential operator defining the equation (5.12) admits the following factorization:

$$
\begin{gathered}
\left(1024 x^{6}-46656\right) \frac{d^{6}}{d x^{6}}+27648 x^{5} \frac{d^{5}}{d x^{5}}+242816 x^{4} \frac{d^{4}}{d x^{4}}+ \\
818944 x^{3} \frac{d^{3}}{d x^{3}}+955780 x^{2} \frac{d^{2}}{d x^{2}}+236180 x \frac{d}{d x}-6545= \\
\left(\left(32 x^{3}-216\right) \frac{d^{3}}{d x^{3}}+432 x^{2} \frac{d^{2}}{d x^{2}}+1526 x \frac{d}{d x}+1309\right) \circ \\
\left(\left(32 x^{3}+216\right) \frac{d^{3}}{d x^{3}}+144 x^{2} \frac{d^{2}}{d x^{2}}+86 x \frac{d}{d x}-5\right) .
\end{gathered}
$$




\section{REFERENCES}

[1] A. Adolphson. Hypergeometric functions and rings generated by monomials, Duke Math. J. 73 (1994), 269-290.

[2] F. Beukers and G. Heckman. Monodromy for the hypergeometric function ${ }_{n} F_{n-1}$, Invent. Math. 95 (1989), 325-354.

[3] J.-E. Björk. Rings of Differential Operators, North. Holland Mathematical Library, 1979.

[4] E. Cattani, C. D'Andrea, and A. Dickenstein. The A-hypergeometric system associated with a monomial curve, Duke Math. J. 99 (1999), 179-207.

[5] A. Dickenstein, L. Matusevich, and E. Miller. Binomial D-modules, manuscript 2006.

[6] A. Dickenstein, L. Matusevich, and T. Sadykov. Bivariate hypergeometric D-modules, Adv. in Math. 196, no. 1, 78-123.

[7] K. G. Fischer and J. Shapiro. Mixed matrices and binomial ideals, J. Pure Appl. Algebra, 113 (1996), 39-54.

[8] I. M. Gel'fand, M. M. Kapranov, and A. V. Zelevinsky. Hypergeometric functions and toric varieties, Funktsional. Anal. i Prilozhen. 23(2) (1989), 12-26.

[9] M. Kato and M. Noumi. Monodromy groups of hypergeometric functions satisfying algebraic equations, Tohoku Math. J. 55 (2003), 189-205.

[10] $\mathrm{Hj}$. Mellin, Résolution de l'équation algébrique générale à l'aide de la fonction $\Gamma$, C.R. Acad. Sc. 172 (1921), 658-661.

[11] K. Mayr. Über die Auflösung algebraischer Gleichungssysteme durch hypergeometrische Funktionen, Monatshefte für Mathematik und Physik 45 (1937), 280-313.

[12] M. Passare, T.M. Sadykov, and A.K. Tsikh. Nonconfluent hypergeometric functions in several variables and their singularities, Compos. Math. 141 (2005), no. 3, 787-810.

[13] M. Passare and A. Tsikh. Algebraic equations and hypergeometric series, in the book "The Legacy of N.H. Abel," Springer-Verlag (2004), 563-582.

[14] T. Sadykov. On the Horn system of partial differential equations and series of hypergeometric type, Math. Scand. 91 (2002), 127-149.

[15] M. F. Singer. Testing Reducibility of Linear Differential Operators: A Group Theoretic Perspective, AAECC 7 (1996), 77-104.

[16] B. Sturmfels, Solving algebraic equations in terms of $\mathcal{A}$-hypergeometric series, Discrete Math. 210, no. 1-3 (2000), 171-181.

[17] U. Walther, Duality and monodromy reducibility of A-hypergeometric systems, arXiv:math.AG/0508622.

Dto. de Matemática, FCEn, Universidad de Buenos Aires, (1428) Buenos Aires, Argentina.

E-mail address: alidick@dm.uba.ar

Department of Mathematics and Computer Science,

Krasnoyarsk State University, 660041, KrasnoyarsK, Russia.

E-mail address: sadykov@lan.krasu.ru 